\title{
Comparative Evaluation of Ray Tracing and Diffusion Equation Modeling in Room Acoustics Design of Subway Stations
}

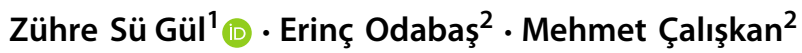

Received: 12 December 2019 / Accepted: 17 February 2020 / Published online: 26 February 2020

(c) Australian Acoustical Society 2020

\begin{abstract}
Due to their disproportionate geometries and uneven distribution of sound, the interior sound field analysis of subway stations has always been challenging. The field necessitates reliable tools for real-case design applications, while the research and discussions on the topic are still ongoing. This study compares two major room acoustics simulation approaches, namely ray tracing and diffusion equation modeling (DEM) in order to assist future acoustical designs of subway stations. Two cases of Istanbul Metropolitan metro lines are selected, namely station BAG with a circular cross section and station USK with rectangular cross section. The reverberation time and relative sound pressure level results from field tests are compared with those obtained by the ray tracing and DEM. The results emphasized the validity as well as limitations of each method over discussed metrics with given geometric layouts and material characteristics of the subway stations.
\end{abstract}

Keywords Subway station · Acoustic design $\cdot$ Diffusion equation $\cdot$ Ray-tracing simulation $\cdot$ Acoustical comfort $\cdot$ Istanbul metro system

\section{Introduction}

Subway or metro systems are integral parts of the substructure within the urban texture. Metropolises and newly developed or growing cities are adding new metro lines to their transport systems each year. Istanbul is one such city, where the population is growing rapidly and traffic is a major problem. While outdoor environmental comfort is one purpose for building underground metro stations, it is equally critical to provide indoor comfort within stations. Considering both health and safety factors, governments have established their own local codes incorporated with international regulations like EU directives for noise management and control in metro stations. As an example, the Turkish Environmental Noise Act of 2010 [1], in compliance with the Environmental Noise Directive (2002/49/EC), specifies the maximum allowable noise levels due to passing trains at the platform edge ( $1.8 \mathrm{~m}$ away) and emergency fan operation with reference to speech intelligibility and optimum rever-

Zühre Sü Gül

zuhre@ bilkent.edu.tr

1 Department of Architecture, Bilkent University, 06800 Ankara, Turkey

2 MEZZO Stüdyo Ltd, 34726 Istanbul, Turkey beration times within stations that should be less than $1.4 \mathrm{~s}$ at mid-frequencies as a design target. All these criteria should be considered during the design phases of stations.

The major reason for the noise pollution in indoor subway stations is the use of durable but highly reflective interior finish surfaces [2]. The geometric proportions of the stations, combined with the reflective tiled or concrete walls and ceilings, result in long reverberation times that have an adverse effect on speech intelligibility of the public announce system [3]. One study [4] highlights that speech intelligibility is better in stations with a rectangular cross section than in stations with an elliptic one, even if the volume of the enclosed space is larger. As the geometry and volume of the stations cannot be varied much, considering the functional and space limitations, the interior surface finishes emerge as the major parameter for the tuning of the indoor acoustic environment. A carefully designed combination of various architectural treatments can significantly improve intelligibility and decrease noise pollution [5-8].

For a smooth design process and successful end product, it is critical to make use of reliable methods in the analysis of station acoustics. The interior acoustical fields of long spaces like metro stations are substantially different from those of proportionate rooms, and this creates a bias in the assumptions of statistical theory [9]. Various methods 
have been proposed for estimating acoustical parameters in long spaces, including wave theory [10], duct theory [11], image-tracing [12, 13], ray-tracing [14], diffusion equation modeling $[15,16]$ and transport equation methods [17-19]. Nevertheless, there is a growing concern among researchers about the theoretical modeling of subway stations as these different methods have different case-specific limitations.

The image source method fails to include diffuse reflections, which is critical when studying sound fields in long enclosures. The ray-tracing method is more flexible than the image source method and more suitable for modeling spaces of complex geometry. The basic limitation of ray tracing is that the method is more valid when the sound field is closer to a diffuse field. The ray-tracing-based methods are able to consider specular and diffuse reflections by making certain assumptions about diffusion. However, some important reflection paths can still be missed in configurations composed of multiple connected spaces. In addition, high absorption in specific surfaces tends to overestimate the sound levels as the distance from the source increases. This specific problem is discussed in Redmore's [20] study on rectangular corridors. His explanation for this overestimation is that for highly absorbent boundaries, the exclusion of diffusion- and angle-dependent absorption is unreasonable. In other words, the assumption of geometrical reflection might be inapplicable.

As another prominent method, by the application of diffusion equation model in room acoustics, the solutions can be reached analytically for simple geometries and numerically for complex enclosures. The speed of computation is much higher for the diffusion model than for ray tracing, especially for complex geometries, and this constitutes one of its basic advantages [21-23]. Ollendorff [24] was the first to develop a theoretical method to calculate noise levels and reverberation times in rectangular road tunnels using the partial differential equation of the diffusion of phonons. The method was too complicated to be used practically at the time, but subsequent work is based on this innovative study. Kuttruff [25] used approximate solutions of the integral equation to simulate the reverberation in long rectangular rooms and proved theoretically that with diffusely reflecting boundaries, the sound attenuation along the length is greater than with geometrically reflecting boundaries. Picaut et al. [15] proposed a theoretical model to describe sound propagation in long rooms with diffusely reflecting boundaries. In their study, simple relations, depending on the perimeter of the cross section and the cross-sectional area, are derived to predict sound attenuation and reverberation times in corridors.

The diffusion equation model (DEM) has been developed further and applied to long rooms by Valeau et al. [16]. One concern about the application of DEM to room acoustics is that in cases with highly absorbent surfaces or boundaries, the assumption of diffusion is contradicted. Following to that,
Billon et al. [26] have performed several studies on cubic rooms and researchers have proposed different absorption factors. Jing and Xiang [27] defined a modified absorption factor for DEM that can model mixed boundary conditions for all room types including cubic rooms, flat rooms and long rooms. The authors tested different absorption coefficients over room surfaces ranging between 0.1 and 0.9 , while validating their model.

Visentin et al. [28] modeled energetic quantities by the diffusion model and by the particle-tracing numerical code in long rooms. In this case, a spatially varying diffusion coefficient for non-homogeneous diffusion is defined. According to this study, the rate of increase in the estimated diffusion coefficient depends on the cross-sectional area of the room and absorption characteristics of the boundaries. The proposed effective diffusion coefficient (Dest) increases linearly with the distance from the source and with decreasing crosssectional area. The proposed model is tested with scale model experiments using a simplified geometry. The concern at this point is that the diffusion coefficient is not known beforehand, when needed in design phase, but obtained after the intensities are already known within the enclosure. Such an empirical study is not practical in real stations with complex geometries, which is a vital focus of this research on the analysis of different methods and their applicability in practice.

Finally, the transport theory [17] is tested in the context of urban canyons for small street widths and very diffuse reflections. As it is difficult to solve complex structures with multiple independent variables, Jing et al. [18] initially developed a one-dimensional transport equation model for simplified cases focusing mainly on sound energy propagation in the "long" dimension. One advantage of the transport equation over DEM is that it can handle explicitly partial specular and partial scattering reflections [19]. Another study by Navarro et al. [29] is based on the acoustic radiative transfer model, which uses the principles of acoustic diffusion equation and the room acoustic rendering equation. The authors conclude that systematic experimental validation is still necessary in order to analyze the reliability of the acoustic radiative transfer equation in room acoustic simulations. In their overview of different simulation techniques in room acoustics, Navarro and Escolano [30] emphasize that the diffusion equation model is an efficient and accurate way of simulating acoustic energy propagation with low computational loads. They stress that in some scenarios, such as disproportionate rooms or coupled-room systems, classical methods and even ray tracing have been proved to be unsuccessful and that some methods are prohibitive as of finite-difference time domain.

To sum up the technical literature, it can be stated that most of the studies of different theories and techniques rely for their validation on simplified geometries of long 
rooms, predominantly entailing rectangular cross sections and highly reflective interior boundaries. Typically, these simplified geometries represent single-track subways. The application of the theories to long rooms has involved purely analytical, one-dimensional analyses. In actual underground urban transport systems, however, there is not just one long dimension, but two or even three tracks or tunnels coupled to each other by branching corridors or larger halls. For such cases, some of the models may become computationally overwhelming. Most of the previous research suggests that further investigations need to be made, in which the diffusion model is applied in more complex geometries, and that the numerical results need to be compared with field measurements, as most of the theoretical validations of long rooms lack full-scale field tests.

Among all reviewed, two most prevailing techniques, namely ray tracing and diffusion equation model (DEM), are investigated further in this study. Real-size measurements are utilized alongside in order to support the comparative evaluation. The major objective is on the discussion of limitations and advantages of selected methods for room acoustics analysis of subway stations as a contribution to the provision of sustainable acoustical design process. Two subway stations are selected for detailed analysis: one with a circular cross section and the other with a rectangular cross section. Both stations are on the Üsküdar-Ümraniye line in the Istanbul Metropolitan metro system as detailed in the following sections.

\section{Research Methods}

This study applies two different theoretical methods: raytracing simulations and diffusion equation model (DEM) analysis. Of the two stations at the one with a circular cross section (station BAG), the acoustical models are tuned using the specifications of the applied materials as well as the results of field tests. The station with the rectangular cross section (station USK) has identical surface finish materials, so the materials absorption data obtained from the initial assessment of BAG are considered to be valid for the USK station as well.

\subsection{Field Measurements}

Field tests were conducted at the station BAG on April 8, 2018 , from 00.30 a.m. to 03.00 p.m., at a time when the station was empty and no trains passed by. To estimate the basic parameters specifying the sound field, the room impulse responses were collected. The equipment is in accordance with ISO 3382-1.35. A B\&K (Type 4292-L) standard dodecahedron omni-directional sound source was used for acoustic excitation driven by a B\&K (Type 2734-A) power amplifier. The room impulse responses at various measurement points were captured by a B\&K (Type 4190ZC-0032) microphone. The sampling frequency of the recorded multispectrum impulse is $48 \mathrm{kHz}$. The DIRAC Room Acoustics Software Type 7841 v.4.1 was used to generate noise signals (e-sweep). The height of the omni-directional sound source was $1.5 \mathrm{~m}$ off the floor, and the microphone height was kept at $1.2 \mathrm{~m}$. Tests were conducted for one source (S) and 14 receiver (R1-R14) positions distributed through both tracks of passenger platforms (Fig. 1). Receiver positions R1-R7 have direct sight lines to the source, whereas receiver positions R8-R14 are not in the direct sound field of the sound source.

\subsection{Ray Tracing}

For the purpose of acquiring impulse responses in a raytracing software, simplified acoustical models of station BAG and station USK are generated via 3D modeling software. The total length of platform in station BAG is $140 \mathrm{~m}$ and the overall width of the platform level is $37 \mathrm{~m}$, while the total length of platform in station USK is $150 \mathrm{~m}$ and the overall width of the platform level is $22 \mathrm{~m}$. Station BAG has an estimated acoustical volume of $12,988 \mathrm{~m}^{3}$, and station USK possesses a volume of $14,318 \mathrm{~m}^{3}$. The difference in the volumes of the two stations is not very significant. Both stations have platform screen door system. The major architectural differences between the two stations are that BAG has a circular cross section, while USK has a rectangular cross section. The source and receiver positions used in the ray-tracing simulation of station BAG are identical to the positions used in the field tests. A similar distribution of source and receiver positions is also used for station USK (Fig. 1).

The interior finish materials of the two stations are identical. The walls are cladded with ceramic tiles. The rail-track tunnels are of painted concrete, along with the bare concrete rail-track floors; the platform-floor surfaces are stone tile. The suspended ceiling system of the platform areas consists of perforated metal panels-with $20 \%$ opening ratio. As a backing $25 \mathrm{~mm}, 50 \mathrm{~kg} / \mathrm{m}^{3}$ mineral wool is applied within a $20 \mathrm{~cm}$ air gap behind. The tested sound-absorptive ceiling treatment displays an absorption coefficient of 0.83 in $1 \mathrm{kHz}$, the data of which are obtained from the accredited laboratory test of the suspended ceiling systems' manufacturer. The average sound absorption of the wall and floor surfaces for $1 \mathrm{kHz}$ is 0.03 . Interior views from the ray-tracing models are presented in Fig. 2. The darker (brown) colors in the raytracing model indicate sound-absorptive ceiling surfaces.

According to a recent study [31] among different room acoustic modeling algorithms, there is a systematic overestimation of low-frequency reverberation and a tendency to underestimate the reverberation time above $2 \mathrm{kHz} .1 \mathrm{kHz}$ is a representative mid-frequency octave band for speech signals 


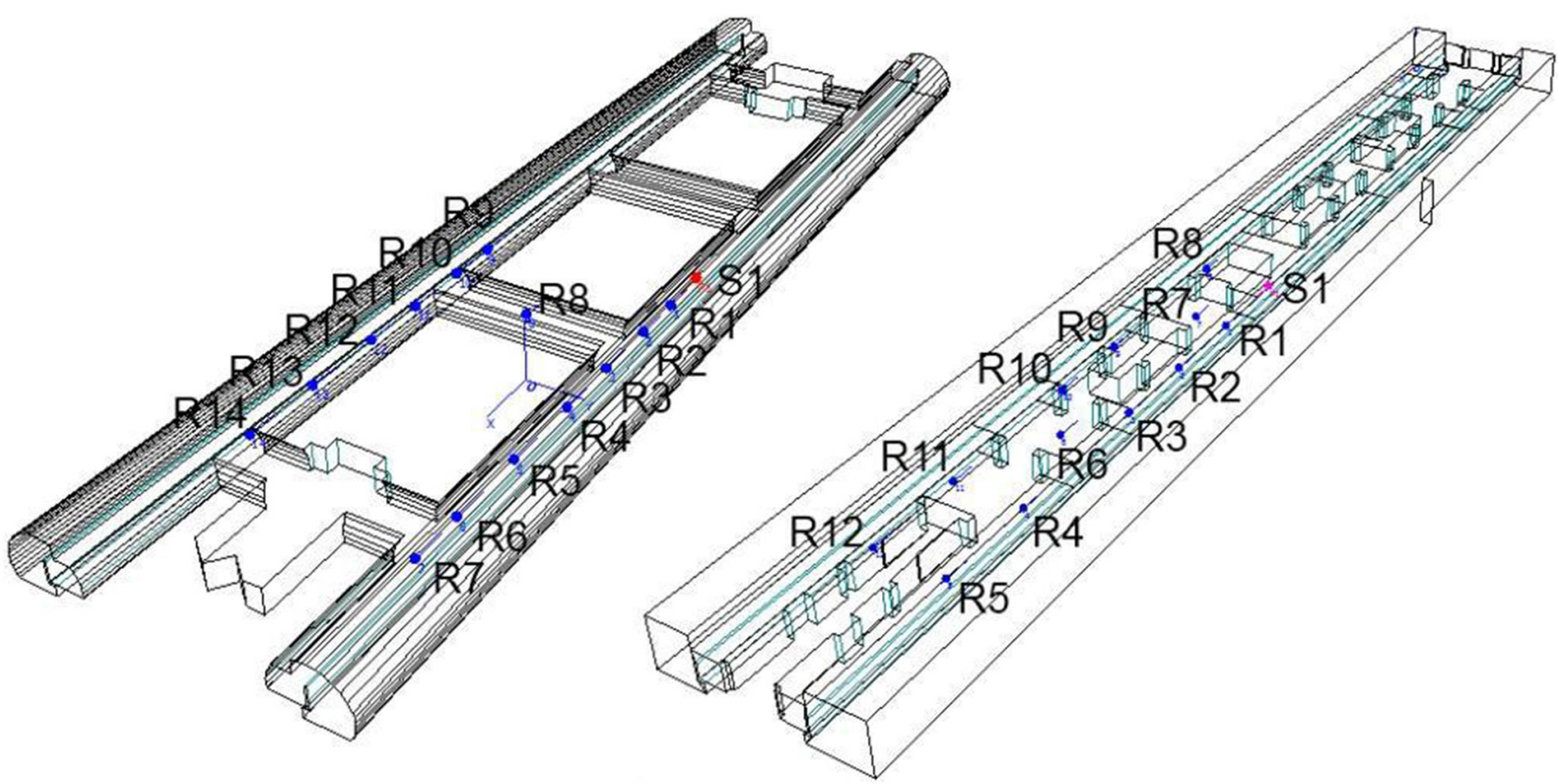

Fig. 1 Source (S) and receiver (R1-R14) positions in acoustical models of station BAG (on the left) and station USK (on the right)
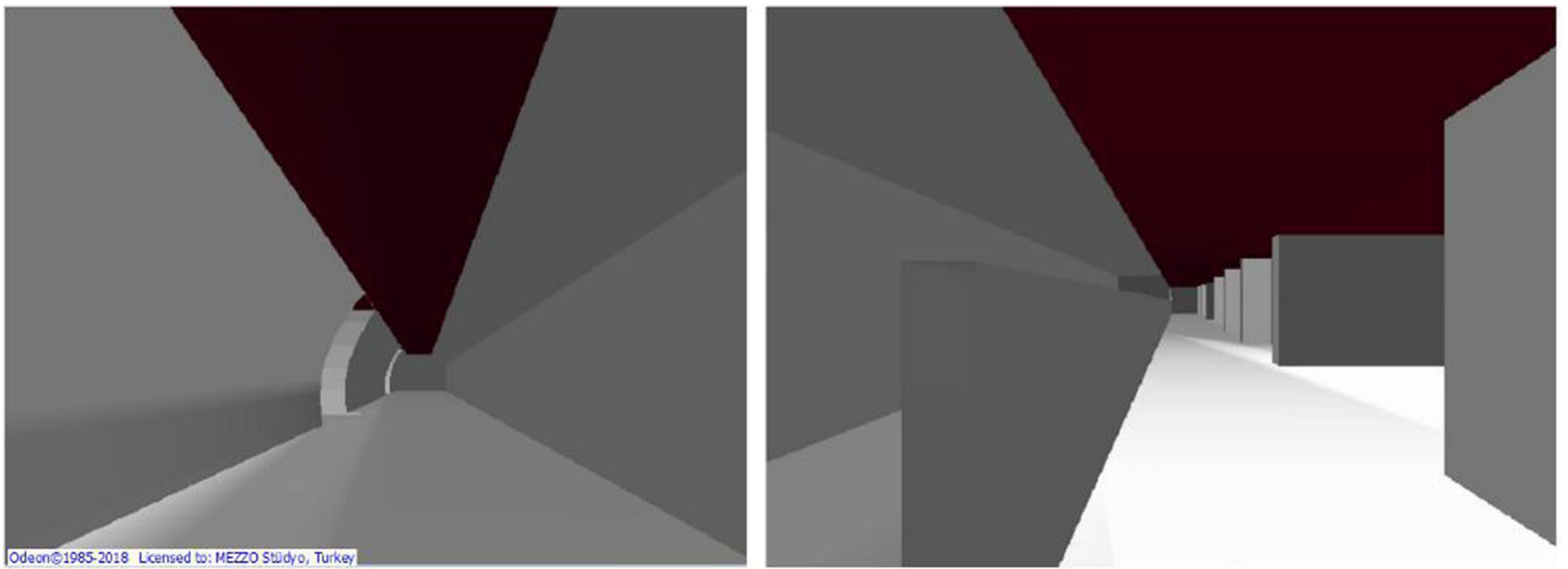

Fig. 2 Interior views from ray-tracing models of platform levels; station BAG (on the left), station USK (on the right)

required to be assessed in regulations, and yet, it is much more reliable for comparisons than low or high frequencies according to the discussion of different theoretical models. For that reason, the comparisons in this paper are given for this specific $1 / 1$ octave band.

It is worth noting that, in ray-tracing simulations, ray numbers reaching up to 500,000 rays are tested in order to provide the sufficient numbers of rays reaching the furthest locations from the source. However, this only increased the mapping time and did not lead to a significant variation in the results. Meanwhile, in order to approximate the ray-tracing solution to the DEM model, scattering coefficients up to 1 are applied.
Different scattering coefficients in ray-tracing models also have not produced a significant variation on the results.

\subsection{Diffusion Equation Modeling (DEM)}

As discussed in the previous literature [15, 16], the DEM is based on the assumption that particles travel along straight lines at the speed of sound in an enclosed space and multiple diffuse reflections occur at the room boundaries, which can be conceived as scattering objects. In order to implement the DEM numerically in a finite element medium, acoustical model of each structure is built. For comparative analysis, exactly the same models and finish materials used in the ray- 


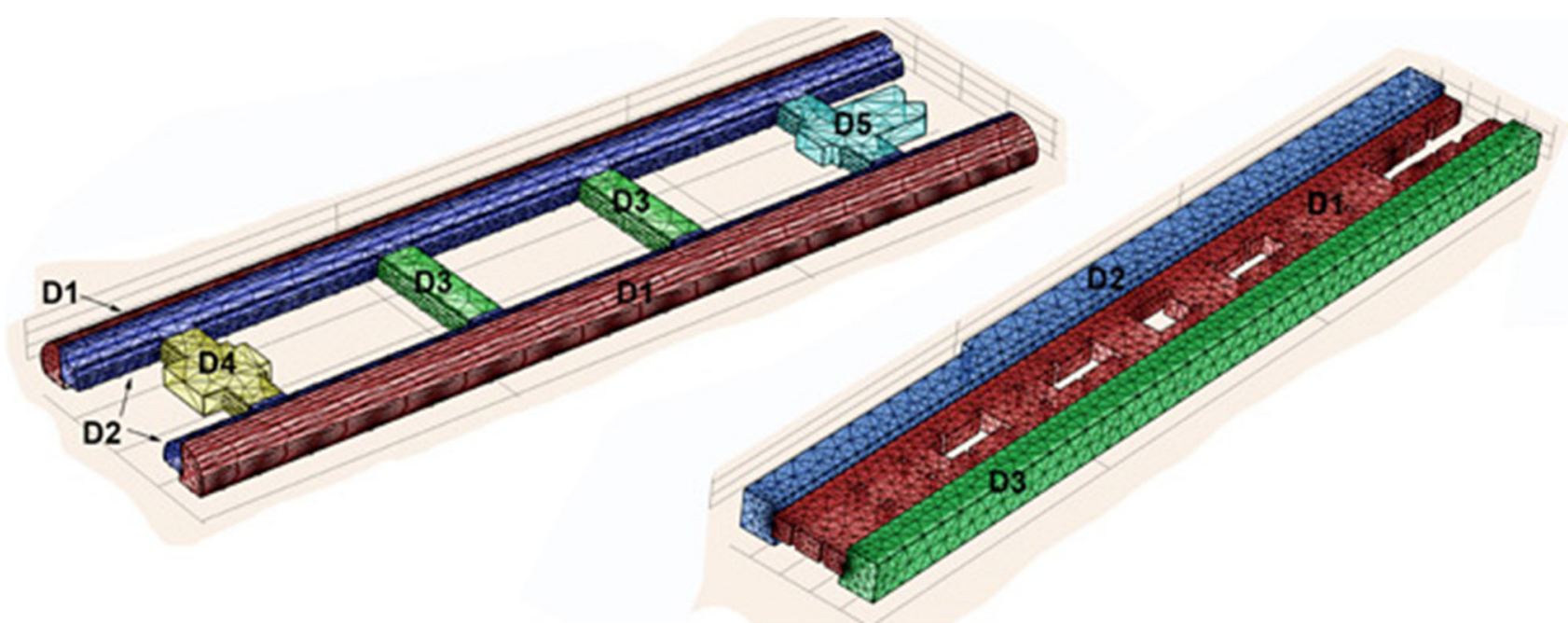

Fig. 3 Mesh models of subway stations: station BAG (below), volume 12,988 $\mathrm{m}^{3}$, total of linear Lagrange-type mesh elements 282,701 , max. mesh size $4.1 \mathrm{~m}$; station USK (above), volume 14,187 $\mathrm{m}^{3}$, total of linear Lagrange-type mesh elements 94,046, max. mesh size $4.9 \mathrm{~m}$

tracing simulations are utilized for the DEM solution of both stations.

One advantage of DEM over other theoretical models, especially in mid-to-high frequencies, is that the meshing size takes its value from $\lambda$, which is the mean free path (MFP). MFP is defined by $4 V / S$, where $V$ is the volume of the space and $S$ is the total surface area. Hence, the meshing size is defined by the room dimensions rather than the wavelength. The only criterion is that as long as the maximum mesh size is smaller than the MFP of the room, then the DEM is applicable. The computational load is therefore lower. The meshed model of station BAG has a total of 282,701 linear, Lagrange-type mesh elements with a maximum mesh size of $4.1 \mathrm{~m}$, whereas the meshed model of station USK has a total of 94,046 linear, Lagrange-type mesh elements with a maximum mesh size of $4.9 \mathrm{~m}$ (Fig. 3). Thus, the maximum mesh sizes of both BAG and USK are smaller than their estimated MFPs, so the DEM is applicable (Table 1). In Fig. 3, different domains for a multi-domain solution are presented. It should be noted that, for the ease of computations, domains are grouped in line with their architectural similarities, ending up with an identical MFP. So those having exactly the same volumetric, form and material features are coded with the same domain number.

\subsubsection{Interior Diffusion Equation}

In a room region or domain $(V)$ with time-dependent energy density $(w)$ at a position $(r)$ and time $(t)$, the sound energy flow vector $(J)$ caused by the gradient of the sound energy density can be expressed by Fick's law [32]:
Table 1 Volume $(V)$, surface area $(S)$, mean free path (MFP) and diffusion coefficient $(D)$ information for single- and multi-domain scenarios (D1-D5) of station BAG and station USK

\begin{tabular}{lrrll}
\hline & $V\left(\mathrm{~m}^{3}\right)$ & $S\left(\mathrm{~m}^{2}\right)$ & MFP $(\mathrm{m})$ & $D$ \\
\hline BAG & & & & \\
Single domain & 12,988 & 11,143 & 4.7 & 533 \\
D1 & 3575 & 2995 & 4.8 & 545 \\
D2 & 2084 & 2810 & 3.0 & 340 \\
D3 & 368 & 393 & 3.8 & 429 \\
D4 & 504 & 521 & 3.9 & 442 \\
D5 & 691 & 694 & 4.0 & 455 \\
USK & & & & \\
Single domain & 14,187 & 11,250 & 5.0 & 577 \\
D1 & 4274 & 4674 & 3.7 & 418 \\
D2 & 5419 & 3875 & 5.6 & 640 \\
D3 & 4459 & 3548 & 5.0 & 575 \\
\hline
\end{tabular}

$J(r, t)=-D \nabla w(r, t)$

where $D$ is the diffusion coefficient, which takes into account the room morphology via its mean free path $(\lambda)$ given by

$D=\frac{\lambda c}{3}=\frac{4 V c}{3 S}$

where $V$ is the volume of the room, $S$ is the total interior surface area of the room and $c$ is the speed of sound. The sound energy density $w$ in a domain $(V)$ changes per unit time as 
$\partial w(r, t) \partial t=-\nabla J(r, t)=D \nabla^{2} w(r, t), \quad \in V$

where Eq. (1) is used to arrive at the right-hand side of Eq. (3) and $\nabla$ is the Laplace operator. In the presence of an omnidirectional sound source within a domain $(V)$, Eq. (3) has to take the omni-directional sound source, $q(r, t)$, into account

$\frac{\partial w(r, t)}{\partial t}-D \nabla^{2} w(r, t)=q(r, t), \quad \in V$

In Eq. (4), the source term is zero for any subdomain where no source is present. To generate the steady-state-derived sound energy decay, a switch-off signal to the source term at zero time is assigned by:

$q\left(r_{\mathrm{s}}, t\right)=E_{0} \zeta(t)$

where

$\zeta(t)=\left\{\begin{array}{l}1, t \leq 0 \\ 0, t>0\end{array}\right.$

Physically, the sound source, a point source, is turned on for a sufficiently long period of time to establish steadystate field conditions and is then switched off at a time point referred to as 0 . In the numerical implementation, a timedependent solution is required before $t=0$ in order to ensure that the system arrives at the steady state.

\subsubsection{Boundary Conditions}

The effects of enclosing room surfaces can analytically be expressed by boundary equations defined on the boundary surfaces $(S)$. The boundary condition established to include energy exchanges on enclosing surfaces is:

$J(r, t) \cdot n=-D \nabla w(r, t) \cdot n=A_{X} c w(r, t), \quad$ on $S$

Equation (7) shows that sound energy is kept within the room boundaries, with $n$ denoting the surface outgoing normal. There is an exchange coefficient or the so-called modified absorption factor for the boundaries in relation to the specific surface material, which is expressed as follows [27]:

$A_{X}=\frac{\alpha}{4(1-\alpha / 2)}$

where $\alpha$ is the absorption coefficient of the specific surface or boundary. Two other absorption factors are previously defined in diffusion-Sabine and diffusion-Eyring models. Meanwhile, the diffusion equation model with a modified absorption factor proposed by Jing and Xiang [27] is capable of modeling rooms with low absorption, as well as for mixed boundary conditions associated with high absorption for specific room surfaces. For that reason, the boundary equations of this study utilize Eq. (8) for the DEM solution of the subway stations chosen as case studies, which have reflective wall and floor surfaces but an absorbent ceiling. Combining Eqs. (7) and (8) gives the following system boundary equation:

$-D \frac{\partial w(r, t)}{\partial n}=\frac{c \alpha}{4(1-\alpha / 2)} w(r, t), \quad$ on $S$

For different types of multi-domain DEM simulations, the aperture boundary surface at the intersection of two domains can be defined by the following [33]:

$\hat{n} \cdot\left[D_{1} \nabla w\left(r_{\mathrm{b}}, t\right)-D_{2} \nabla w\left(r_{\mathrm{b}}, t\right)\right]=0$

which represents a continuity boundary condition on interior boundaries at the aperture position $r_{\mathrm{b}}$, where $D_{1}$ is the diffusion coefficient in the primary room, and $D_{2}$ is the diffusion coefficient for the secondary room.

In this study, the case subway stations comprise two rail tracks (tunnels) combined to each other by platform corridors or smaller halls. The subway models could either be considered as a single domain working as a single space or as a space with multiple domains coupled to each other. In order to test the strength of this coupling and later choose the reliable approach, the DEM is computed for both singleand multi-domain models. Table 1 lists the volume and the total surface area of individual domains. Accordingly, the mean free paths (MFPs) and diffusion coefficients $(D)$ are calculated for different domains of case stations.

For obtaining the time-dependent solutions of meshed models, Eqs. (4), (5), (9) and (10) are applied where necessary. Sound energy densities $w(r, t)$ 's are then converted into sound level (SL) in decibels as follows:

$\mathrm{SL}=10 \log _{10} w(r, t)$

The time-dependent simulation takes approximately $6 \mathrm{~min}$ $38 \mathrm{~s}$ on a computer with an Intel(R) Xeon(R) E5-1650 CPU and a $3.60 \mathrm{GHz}$ processor for station BAG and $2 \mathrm{~min} 35 \mathrm{~s}$ for station USK DEM computations. There is no significant change in the speed of computation when single- and multidomain solutions are compared. After the model is solved, the sound energy decays or energy flow vectors are easily obtained for any coordinate point within the volume or observed through 3D spatial mapping and slice plots in a finite element post-processing, but not provided within this paper for the sake of briefness. This constitutes an advantage of DEM over ray tracing. 


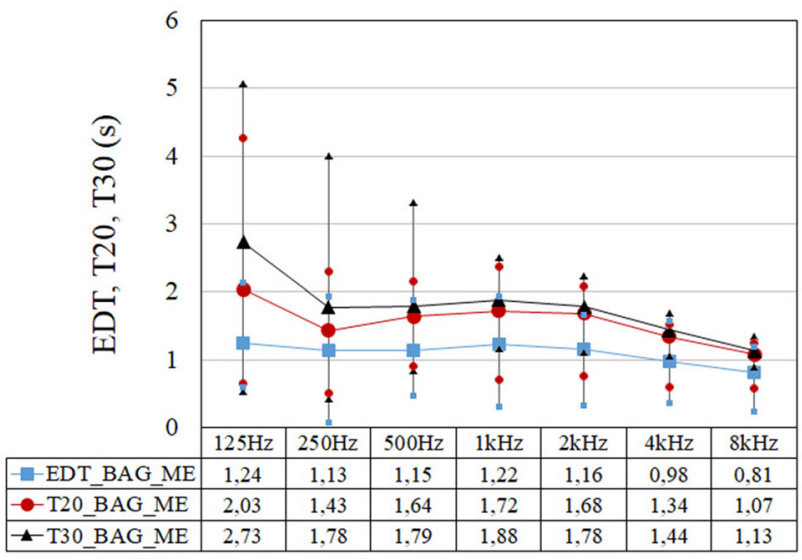

Fig. 4 Station BAG field test (ME) results of EDT, T20 and T30 in octave bands averaged over receiver positions with maximum and minimum values

\section{Results and Discussion}

In this section, the field measurements, the finite element solution of DEM and the ray-tracing simulation results are presented comparatively in an effort to identify the strengths and weaknesses of different room acoustics analysis theories and methodologies for disproportionate subway stations. The field measurement data are provided for station BAG in detail.

\subsection{Field Measurement Results}

Initially, the octave band results of different acoustical metrics are given in order to archive full-scale measurement data that may be of use to some other research on this specific topic. Accordingly, Fig. 4 presents the decay rate parameters of station BAG including early decay time (EDT), T20 and T30 averaged over the receiver positions with the maximum and minimum values. The trend in the decay rates is similar in that there is a reduction in the reverberation time values as the frequency increases. This is a typical outcome due to the sound absorption performance of fibrous materials over frequency. Most fibrous materials are more absorptive at high frequencies, and the performance in low frequencies depends upon the thickness or depth of the air gap behind. In this case, the ceiling surfaces are perforated metal backed with mineral wool within a $20 \mathrm{~cm}$ cavity.

The deviation between the T30 and T20 results reaches a maximum of $0.7 \mathrm{~s}$ at $125 \mathrm{~Hz}$. The values are very close at mid- and high frequencies. The difference between the EDTs and T20 and T30 results is much more pronounced. In the overall frequency range, the differences vary between 0.3 and $1.5 \mathrm{~s}$. In all cases, the EDTs are lower than the T20 and $\mathrm{T} 30$ results. The EDT representing the decay rate in the first $10 \mathrm{~dB}$ drop is much faster than in the $20 \mathrm{~dB}$ and $30 \mathrm{~dB}$ drops

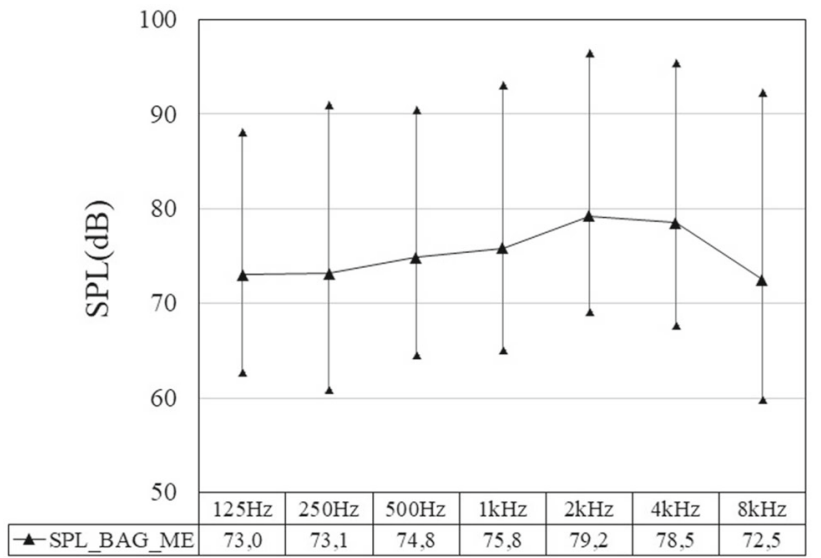

Fig. 5 Station BAG field test (ME) results of SPL in octave bands averaged over receiver positions with maximum and minimum values

in the decay curve. The later reflections or later reverberance is much more dominant in T20 and T30, whereas the nearby surfaces are more influential in the EDT results. Accordingly, the deviations over the averaged receiver positions distributed throughout the station, as observed from the minimum and maximum values, are larger in the EDT plot than in the T20 and $\mathrm{T} 30$ results.

Figure 5 displays the station BAG field test results for SPL over octave bands averaged for receiver positions with maximum and minimum values. The SPL displays a flat pattern, without significant shifts, over the frequency interval between $125 \mathrm{~Hz}$ and $8 \mathrm{kHz}$. The values increase slightly toward $4 \mathrm{kHz}$ and drop at $8 \mathrm{kHz}$. The drop of SPL in $8 \mathrm{kHz}$ relates to the air absorption. The changes in the SPL values over the frequency spectrum are both dependent on the sound power distribution of the source signal, the background noise content, the air volume and the absorption area of surface materials. The deviations of SPL values between receiver positions are in the interval of $20-30 \mathrm{~dB}$. This is acceptable considering that the SPL values drop as the source-receiver distance increases, especially when the sound field is not diffuse. Another reason is that some receiver positions are in the shadow zone, whereas some have direct sound arrival in addition to the early reflections.

\subsection{Comparative Analysis of Measurements Versus Simulations}

\subsubsection{Station BAG}

The second part of discussion in this study constitutes the comparative analysis of field test, DEM computations and ray-tracing simulation results. Figure 6 compares the field test, single- and multi-domain DEM and ray-tracing results of EDT, T20 and T30 in station BAG for receiver positions $\mathrm{R} 1-\mathrm{R} 14$ at $1 \mathrm{kHz}$. As described previously, the reliability 
of the DEM models for both stations is checked by singledomain and multi-domain model comparisons. According to Fig. 6, the decay rates of the multi-domain DEM solution are 0.2-0.4 s higher than those of the single-domain DEM solution. On the other hand, the single-domain DEM results are much closer to the field test results than the multi-domain DEM results. This means the BAG station space does not act as a coupled-volume system and that the apertures connecting different volumes (the halls/inter-connecting tunnels and the two main passenger platform tunnel) to each other are not small enough to cause non-exponential sound energy decay as previously observed in different structures [34]. Thus, the single-domain solution of station BAG is more reliable than its multi-domain solution. So, the discussion is held for single-domain solutions of station BAG afterward.

The single-domain DEM results for decay rates are much lower than the ray-tracing results. For the same model with the same materials assigned, the ray-tracing decay rate results, except for EDT in short source-receiver positions, are 0.9-1.0 s higher than the single-domain DEM results. The T30 values obtained from the single-domain DEM for receiver positions R3, R4 and R5 are almost identical to the field test results. As the distance increases, the deviation ranges between 0.1 and $0.3 \mathrm{~s}$. This deviation is much smaller than the difference observed when the ray-tracing results are compared to the field test results. The ray-tracing results are at least $1.0 \mathrm{~s}$ higher than the field results for all receiver positions. In short source-receiver distances including R1 and R2, the DEM results are $0.5 \mathrm{~s}$ higher than the field test results.

The divergences between the field test results and the other simulation methods increase in T20 and peak at EDT. This means that T30 is a much more reliable basis of assessment in the case of subway stations for both simulation methods. On the other hand, EDT is more prone to early reflections than T20 and T30, while DEM is more reliable for the portion of the decay that is mostly affected by late reflections. This might be one reason why the EDT results deviate more when compared to the T30 results in the DEM versus field results comparison.

Overall, the single-domain decay rate DEM results are highly correlated with the field test results. Ray tracing overestimates the decay rates, and there are abrupt jumps in reverberation times at different receiver positions, when they are compared to the field test results. Both the field test results and the simulated decay rates indicate that the reverberation increases as the distance from the source increases (Fig. 6). This outcome was also previously observed by Kang [13], who highlighted the fact that in long spaces such as metro stations, the reverberation time is very dependent on the distance between source and receiver and cannot be assumed to have a single value for the entire space, thus also proving the inadequacy of statistical theory in disproportionate rooms.

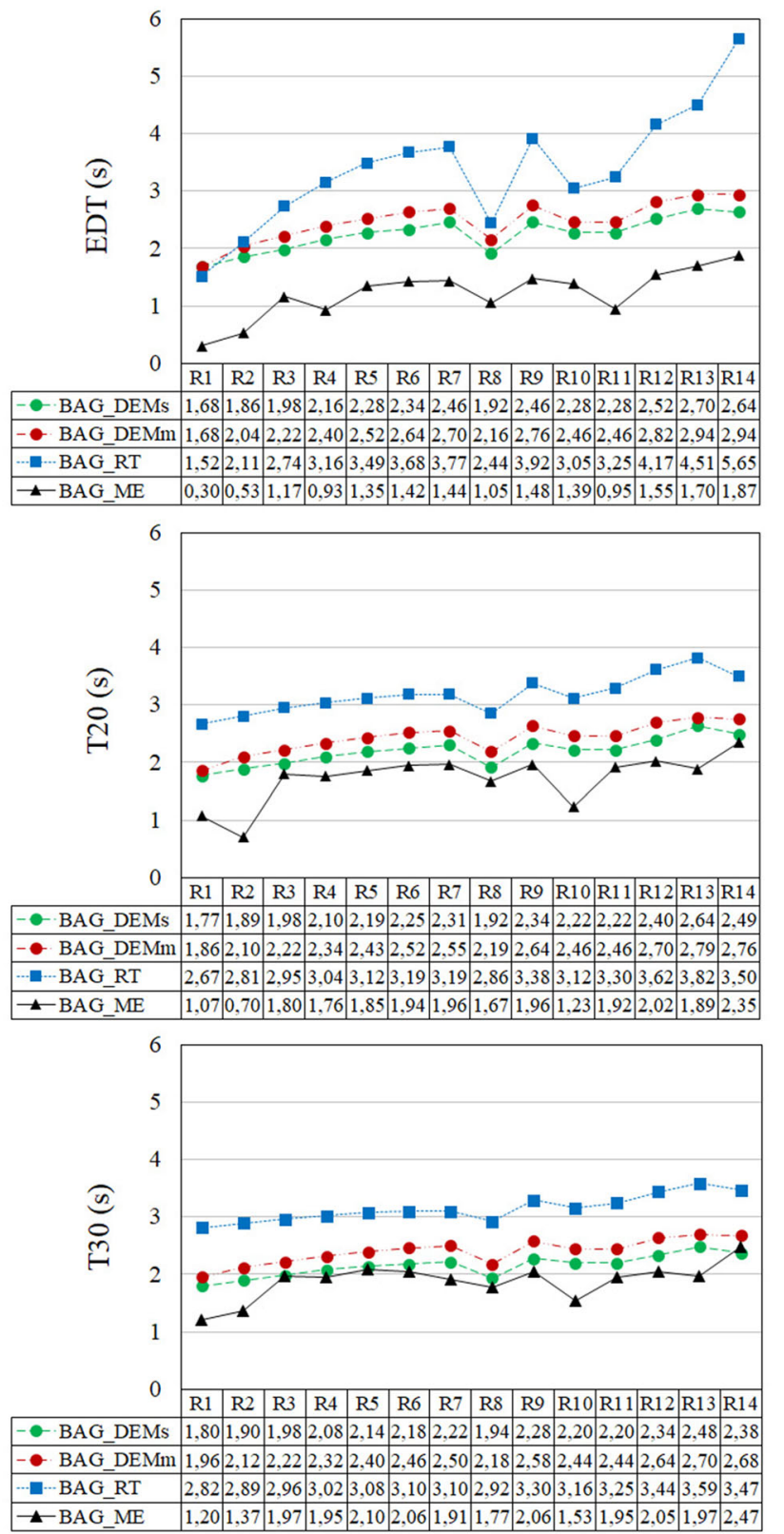

Fig. 6 Comparison of field test (ME), single (s)- and multi-domain (m) DEM and ray-tracing (RT) results of EDT, T20 and T30 in station BAG for different receiver positions (R1-R14) at $1 \mathrm{kHz}$

Figure 7 compares the relative sound pressure levels (SPLs) measured in station BAG at different receiver positions (R1-14) at $1 \mathrm{kHz}$, according to the single-domain DEM, multi-domain DEM and ray-tracing results. In the comparison of the field data and the results of ray tracing and DEM, the relative SPL distributions in reference to $\mathrm{R} 1$ - that is, the closest receiver position to the sound source-are considered, since the absolute values of SPL are dependent on the source power and might bias the results. The results of all the tests and simulations indicate, as expected, that the relative sound level values drop as the distance between source and 


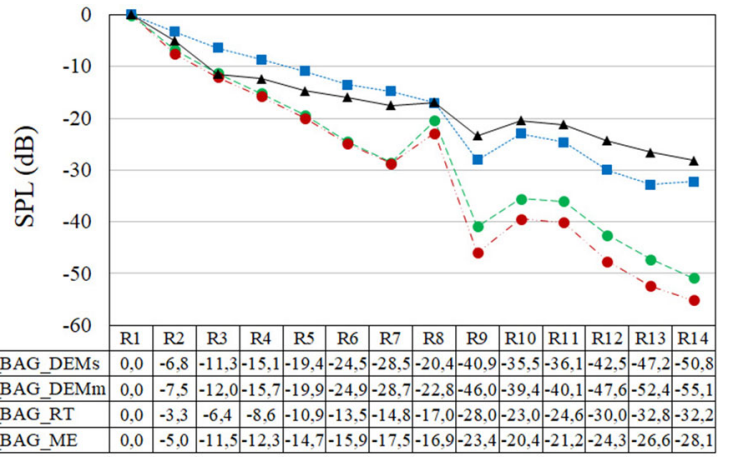

Fig. 7 Comparison of field test (ME), single (s)- and multi-domain (m) $\mathrm{DEM}$ and ray-tracing $(\mathrm{RT})$ results of relative sound level $(\mathrm{dB})$ in station BAG for different receiver (R1-14) positions for $1 \mathrm{kHz}$

receiver increases. R8, as shown in Fig. 1, is located in the connection tunnel and is actually much closer to the source in comparison with R5 and R7, but does not have a direct sight to the sound source. The increase in level at that particular receiver position is therefore rational.

When the different methods are compared, in contrast to the findings for decay rates, relative sound levels turn out to be closer to the field test results in the case of ray tracing than in the DEM solutions, especially as the source-receiver distances increase. Over short distances including R2 and R3 - that is, at most $20 \mathrm{~m}$ away from the sound source-the DEM results are much more compatible with the field tests, whereas the attenuation of sound energy shown by ray tracing is less than indicated by the field data. After that point, however, the deviation between the field results and the DEM results increases.

In station BAG, the single-domain DEM solution is much more consistent with the field data than the multi-domain DEM solution for the SPL parameter as well. When the single-domain DEM results are compared with the measured data, the deviation starts at $3 \mathrm{~dB}$ for the closest positions and reaches a maximum of $20 \mathrm{~dB}$ at the furthest receiver location. In the case of ray tracing, the divergences from the field data are around $4-5 \mathrm{~dB}$. Thus, it can be concluded that the DEM method overestimates the relative degree of sound attenuation more as the source-receiver distance is increased. This outcome implies that with diffusely reflecting boundaries, as in the case of the diffusion model, the sound attenuation along the length is greater than the one with specularly reflecting boundaries. The physical explanation of this, as Kutruff [25] previously stated, is that with diffusely reflecting boundaries, the sound rays have more chances of impinging upon the boundaries. In this case, the diffusion approach within DEM still causes a greater attenuation of sound levels in comparison with the ray-tracing theory, as the distance from source to receiver increases.

\subsubsection{Station USK}

Figure 8 compares the single- and multi-domain DEM and ray-tracing results of EDT, T20 and T30 in station USK for receiver positions R1-R12 at $1 \mathrm{kHz}$. Overall, the multidomain DEM decay rate results are 0.1-0.2 s higher than the single-domain DEM solution. This slight variation is even smaller than the deviations observed in station BAG. This is due to the fact that station USK is even less fragmented than station BAG (Figs. 1, 3) and the station space, together with its two track volumes and central platform area, behaves more as a single-space volume. Thus, multi-domain investigation proves not to be necessary in this case either.

In both station BAG and station USK, the trend as the source-receiver distance increases is for the EDT to show a higher increase than the RT. This outcome was previously observed in Valeau's study as well [16]. On the other hand, the early part of sound decay is more affected by the nearby room surfaces in a ray-tracing simulation than in its DEM solution, while the DEM results are still much closer to the field data. The single-domain DEM results for decay rates in the case of station USK are again around $1.0 \mathrm{~s}$ lower than the ray-tracing results, as in the case of station BAG. The distribution pattern over receiver positions for both $\mathrm{T} 20$ and T30 is very similar in both simulation methods. The deviations in EDT results over receiver positions in ray tracing are larger, as is observed in the station BAG ray-tracing results for EDT when compared with the field test results and DEM solutions.

In general, for station USK there is a slight increase in $\mathrm{T} 20$ and T30 in the range of $0.3-0.4 \mathrm{~s}$ for single-domain DEM and $0.4-0.8 \mathrm{~s}$ for ray tracing, as the source-receiver distance increases. The difference between the maximum and minimum EDT values over receiver positions in ray tracing is almost $3.0 \mathrm{~s}$, whereas for the single-domain DEM solution, it is only $0.7 \mathrm{~s}$. This outcome once again calls into question the overestimation of decay rates by ray-tracing method in long enclosures.

Figure 9 compares the single-domain DEM, multi-domain DEM and ray-tracing results for relative SPLs in station USK for the different receiver positions (R1-12) at $1 \mathrm{kHz}$. In all the simulated results, the SPL values drop as the source-receiver distance increases. The pattern is similar to that for station BAG. R7, as shown in Fig. 1, is located in the middle of the platform area, so it is actually much closer to the source than R2-R5. Similarly, R6, located on the central axis, is closer to the source in comparison with R4 and R5. Thus, the relative drops in SPL by receiver position are expected.

The difference between the single-domain and multidomain DEM results is within $0-2 \mathrm{~dB}$. Once again, this is a very slight discrepancy, as observed in the decay rate results from station USK. For this reason, only the DEM results from the single-domain solution will be discussed from this point. The minimum difference between the results obtained from 


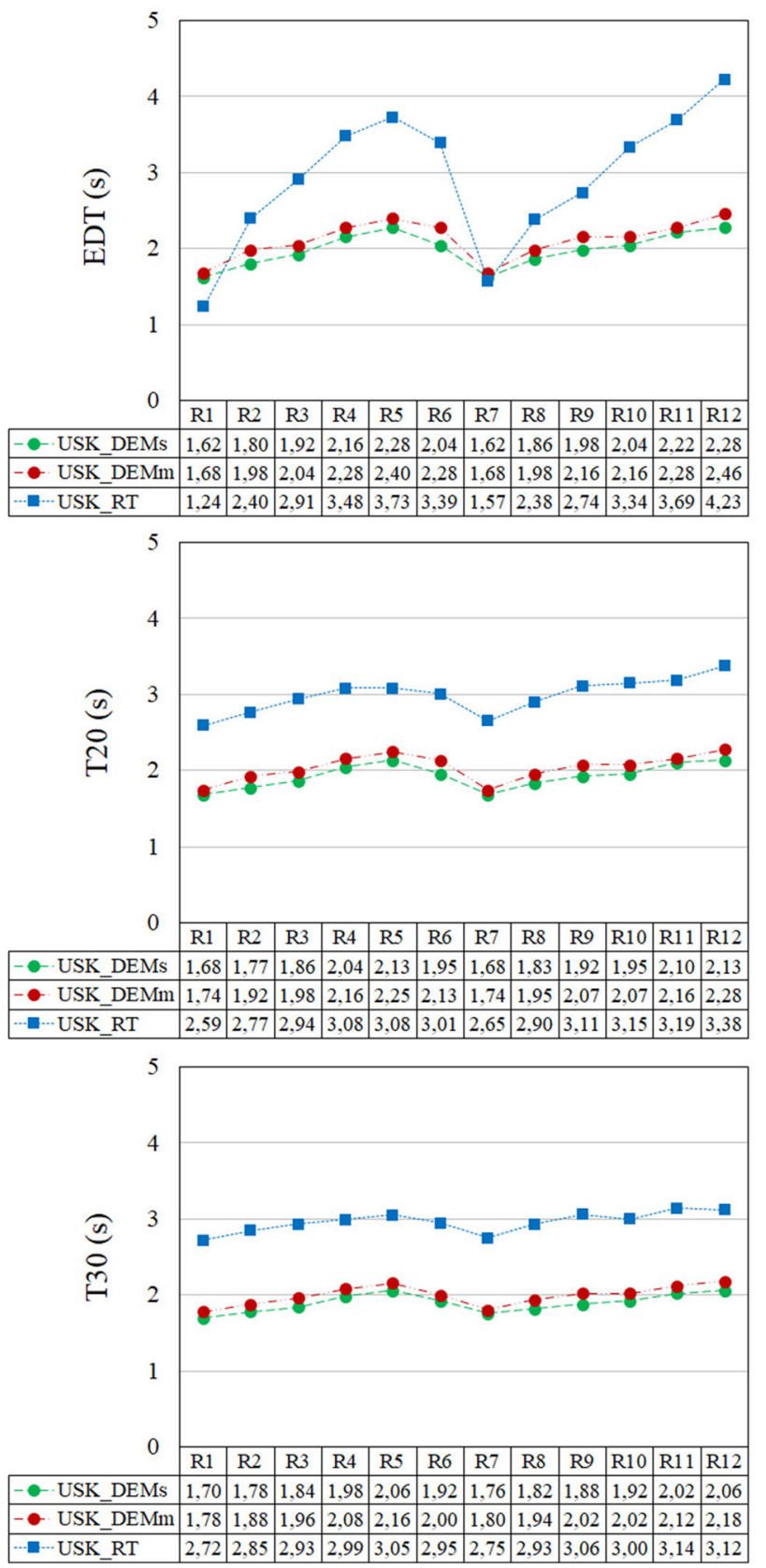

Fig. 8 Comparison of single (s)- and multi-domain (m) DEM and raytracing (RT) results of EDT, T20 and T30 in station USK for different receiver positions (R1-R12) at $1 \mathrm{kHz}$

single-domain DEM and ray tracing is lower than $1 \mathrm{~dB}$ for the receiver positions closest to the sound source, which is not a noticeable difference. The deviation reaches a maximum of $20 \mathrm{~dB}$ at the furthest receiver position which remains in the shadow zone of the sound source. Thus, the DEM solution shows the relative sound attenuation in station USK, too, to be higher than suggested by the ray-tracing results. In summary, the general trend that emerges from the comparison of the results of ray tracing with the results of the DEM method

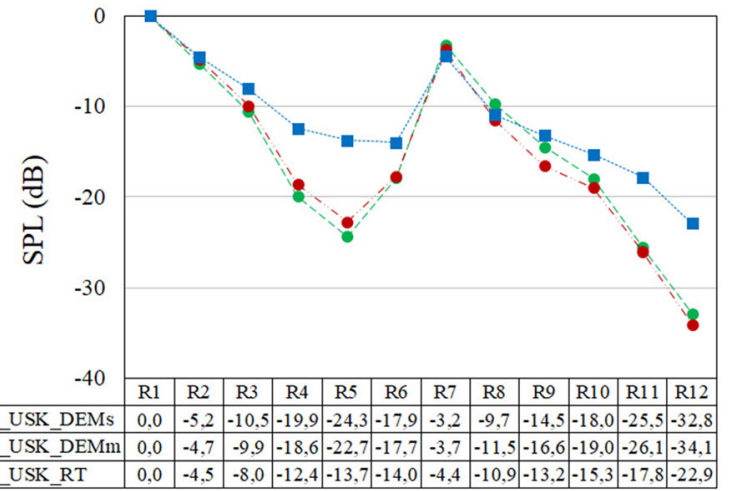

Fig. 9 Comparison of single (s)- and multi-domain (m) DEM and raytracing (RT) results for relative sound level $(\mathrm{dB})$ in station USK for different receiver positions (R1-12) at $1 \mathrm{kHz}$

in station USK in regard to both the SPL and decay rate parameters is similar to the trend observed in station BAG.

\subsubsection{Distribution Maps}

Finally, at $1 \mathrm{kHz}$ relative SPL distribution map for overall passenger platform surface has been obtained for station BAG (Fig. 10) using both the ray-tracing simulations and the single-domain DEM computations. When the ray-tracing results for sound attenuation are compared to the DEM results, it can be seen that there is a $15-20 \mathrm{~dB}$ difference in attenuation for the furthest receiver (or grid) locations, with ray tracing showing lower attenuation values. These furthest locations correspond to the end of tunnels, which are assigned to be absorptive, as in the real case of continuous boundary, and thus, interfering end reflections are prohibited in both models. On the other hand, the connection tunnels between tracks as well show a similar trend in both models. The major difference between DEM and ray tracing as can be followed from relative levels in each grid location is that the overall attenuation is greater for DEM than it is for the ray tracing. The relative sound attenuation values obtained by ray tracing are closer to the measured data than the DEM results in the case of long source-receiver distances, as shown in Fig. 7, whereas for short source-receiver distances, the relative SPL attenuation values obtained by DEM are much better matched with the field data. This outcome indicates that DEM computation is much more suitable than ray tracing when criteria in relation to short source-receiver distances are being assessed. For estimating acoustical parameters at greater source-receiver distances in long dimension, which is after $20 \mathrm{~m}$ in this particular case, ray tracing is more reliable. This distance is almost six times longer than the shortest room dimension, $3 \mathrm{~m}$, which is the suspended ceiling height. On the other hand, the reverberation times and variations in 


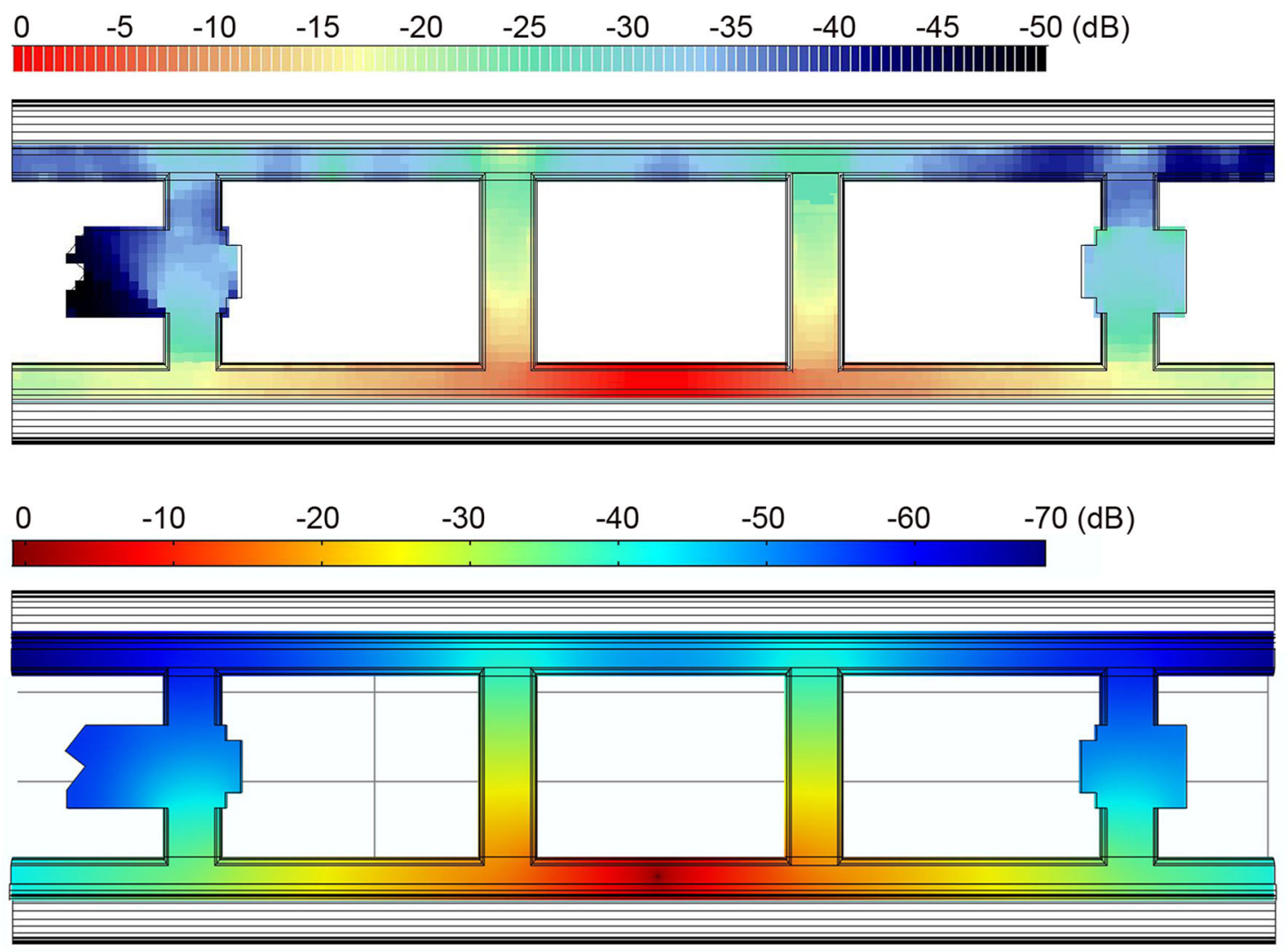

Fig. 10 Comparison of single-domain DEM (below) and ray-tracing (above) maps of relative sound level (dB) estimated for station BAG at $1 \mathrm{kHz}$

decay rates obtained from DEM are more compatible with the field data than those obtained by the ray-tracing method.

\section{Conclusion}

The optimization of acoustical comfort parameters in subway stations is a major concern in their acoustical design. Absorptive materials- "acoustical materials" in common parlance-are generally much more expensive than standard building materials. The use of unnecessary quantities of absorptive materials is not a sustainable solution. Recent feedback on various constructed subway stations has drawn attention to the overuse of acoustical materials. Motivated by this concern, the present study has revisited different theories and methods for estimating sound fields in subway stations, which generally have long and disproportionate room ratios. Field tests provide good feedback for future designs, while every station is specific and has to be designed individually. The reliable optimization of the use of absorptive materials in fulfilling acoustical design criteria requires the application of reliable analysis methods during design phases.

Much research has been undertaken on long enclosures for the sake of proposing the most reliable acoustical estimation tools. Different methods have turned out to display different advantages and drawbacks as discussed in this paper. Among all the methods, the ray tracing and the diffusion equation model (DEM) are found to be the two most prevalent methods for disproportionate spaces, but there was still ambiguity on the advantages and drawbacks of the two models. On the other hand, in contrast to ray-tracing technique the application of the DEM was mostly limited to theoretical research rather than real-case design studies.

Most studies confirmed their findings over simplified geometries in the form of single-track corridors or tunnels. However, the propagation and attenuation of sound in reallife long enclosures are more complicated. Scale models are frequently applied for experimentation, while theoretical studies still need to be validated through full-scale acoustical measurements. The previous literature contains only a limited amount of field measurements. Besides, in some studies, tests could not be made for long source-receiver distances due to certain practical limitations, and in some cases, the stations tested did not have an absorptive ceiling. For that reason, comparisons of the full-scale tests in a real complex subway case to most recent approaches in room acoustics simulations are one of the contributions of this study. 
There are two major stimulating findings of this research to emphasize. One of them is that ray-tracing simulations overestimate decay rates and that DEM computation is more consistent with field measurement results. Among different decay rate metrics, T30 patterns obtained by DEM are much closer to the field test results than T20, while EDT is the least consistent. This outcome is directly related to the increasing reliability of the DEM in later reflections or the reverberant field, while EDT is affected by the nearby early reflections. The other outcome, tested through relative sound levels, is that the DEM method shows greater sound attenuation as the distance from the source to the receiver is increased, which are higher than those indicated by the field tests. This result implies that with diffusely reflecting boundaries of DEM, the sound attenuation along the length is greater than if they would be considered as specularly reflecting boundaries. In this respect, the ray-tracing results are more consistent with the field results. These opposing outcomes for the different metrics may be due to the fact that reverberation is determined by multiple late reflections, whereas SPL mainly depends on direct sound and early reflections. As DEM inherently disregards the direct sound and early reflections, the attenuation in SPL appears much greater than the ray-tracing solution indicates. With added absorbers - in this case, the absorptive ceiling surfaces-direct sound is the more dominant factor in relative SPL results obtained by ray tracing, but this is not captured in a DEM solution. On the other hand, the added absorbers have more impact on later reflections in the longer dimension of the room. As DEM is more applicable to the later part of the energy decay or the reverberant tail, it factors in the effect of absorbers more evidently in comparison with ray tracing, and the decay rates become progressively lower and eventually closer to the field test results.

Another part of the arguments is on the discussion of the diffusion coefficient that it may not act as a constant in the case of longitudinal spaces. The problem with the non-homogenous diffusion coefficient is that it contradicts with the potential use of diffusion equation model as the non-homogenous diffusion coefficient is currently relying on empirical results. However, this may be one reason for inconsistency of relative sound levels estimated by DEM at distant locations. On the other hand, reverberation times or decay rates for overall receiver surfaces and sound level values for receiver positions that are closer than $20 \mathrm{~m}$ to the sound source, in this case, gathered out of DEM are still reliable with a constant diffusion coefficient. Most of the criteria in codes and regulations are defined for $500 \mathrm{~Hz}$ and $1 \mathrm{kHz}$ and are mostly specified for the nearby receiver positions to the sound source. For this reason, results obtained from DEM with a constant diffusion coefficient prove their efficiency at such locations. This is an important outcome for emphasizing the potential use of DEM in practice, when the measurement positions in the codes are of priority.
In terms of sound levels, the tendency to show excess attenuation seems to be the lone drawback of DEM in comparison with ray tracing for long source-receiver distances. Train noise and the reliability of line sources in a DEM solution constitute another research question that must be the subject of a future study. One other advantage of DEM over ray tracing is the computational speed, especially when the number of receivers is increased. Considering the acoustical criteria defined in codes and standards, reverberation time is a critical traditional parameter and still accepted as the primary indicator in room acoustics design. The optimization of materials in acoustical design starts with the reverberation time estimations, and in this regard, DEM emerges as a reliable method for use in preliminary design phases of subway stations.

Acknowledgements The authors gratefully acknowledge the subcontractor for engineering services, Prota Engineering Inc., contractor, Doğuş Construction Inc. of Istanbul, and operator, Metro Istanbul, for their collaboration in design process and support in providing permissions for the field measurements.

\section{References}

1. Turkish Environmental Noise Act of 2010, Article 12, Passage 3, Table 5

2. Sü, Z., Çalıskan, M.: Acoustical design and noise control in metro stations: case studies of the Ankara metro system. Build. Acoust. 14(3), 231-249 (2007)

3. Kootwijk, P.A.A.: The speech intelligibility of the public address systems at 14 Dutch railway stations. J. Sound Vib. 193(1), 433-434 (1996)

4. Nowicka, E.: Assessing the acoustical climate of underground stations. Int. J. Occup. Saf. Ergon. (JOSE) 13(4), 427-431 (2007)

5. Kim, Y.H., Soeta, Y.: Architectural treatments for improving sound fields for public address announcements in underground station platforms. Appl. Acoust. 74, 1205-1220 (2013)

6. Shimokura, R., Soeta, Y.: Sound field characteristics of underground railway stations-effect of interior materials and noise source positions. Appl. Acoust. 73, 1150-1158 (2012)

7. Kang, J.: Acoustics of long underground spaces. Tunn. Undergr. Space Technol. 12(1), 15-21 (1997)

8. Kang, J.: Scale modelling for improving the speech intelligibility from multiple loudspeakers in long enclosures by architectural acoustic treatments. Acta Acust. 84, 689-700 (1998)

9. Beranek, L.L.: Acoustics. McGraw-Hill, New York (1954)

10. Davies, H.G.: Noise propagation in corridors. J. Acoust. Soc. Am. 53(1973), 1253-1262 (1973)

11. Doak, P.E.: Fundamentals of aerodynamic sound theory and flow duct acoustics. J. Sound Vib. 28, 527-561 (1973)

12. Li, K.M., Lu, K.K.: Propagation of sound in long enclosures. J. Acoust. Soc. Am. 116(5), 2759-2770 (2004). https://doi.org/10. $1121 / 1.1798351$

13. Kang, J.: A method for predicting acoustic indices in long enclosures. Appl. Acoust. 51(2), 169-180 (1997)

14. Yang, L.N., Shield, B.M.: Development of a ray-tracing computer model for the prediction of the sound field in long enclosures. J. Sound Vib. 229(1), 133-146 (2000)

15. Picaut, J., Simon, L., Polack, J.D.: Sound field in long rooms with diffusely reflecting boundaries. Appl. Acoust. 56, 217-240 (1999) 
16. Valeau, V., Picaut, J., Hodgson, M.: On the use of a diffusion equation for room acoustic prediction. J. Acoust. Soc. Am. 119(3), 1504-1513 (2006)

17. Pollès, T.L., Picaut, J., Bérengier, M.: Sound field modeling in a street canyon with partially diffusely reflecting boundaries by the transport theory. J. Acoust. Soc. Am. 116(5), 2969-2983 (2004)

18. Jing, Y., Larsen, E.W., Xiang, N.: One-dimensional transport equation models for sound energy propagation in long spaces: theory. J. Acoust. Soc. Am. 127(4), 2312-2322 (2010). https://doi.org/10. $1121 / 1.3298936$

19. Jing, Y., Xiang, N.: One-dimensional transport equation models for sound energy propagation in long spaces: simulations and experiments. J. Acoust. Soc. Am. 127(4), 2323-2331 (2010). https://doi. org/10.1121/1.3303981

20. Redmore, T.L.: A theoretical analysis and experimental study of the behavior of sound in corridors. Appl. Acoust. 15, 161-170 (1982)

21. Sü Gül, Z., Xiang, N., Çalışkan, M.: Investigations on sound energy decays and flows in a monumental mosque. J. Acoust. Soc. Am. 140(1), 344-355 (2016)

22. Sü Gül, Z., Xiang, N., Çalışkan, M.: Diffusion equation based finite element modeling of a monumental worship space. J. Comput. Acoust. 25(4), 1-16 (2017)

23. Sü Gül, Z., Odabaş, E., Xiang, N., Çalışkan, M.: Diffusion equation modeling for sound energy flow analysis in multi domain structures. J. Acoust. Soc. Am. 145(4), 2703-2717 (2019). https://doi. org/10.1121/1.5095877

24. Ollendorff, F.: Statistical room-acoustics as a problem of diffusion. Acustica 21, 236-245 (1969)

25. Kuttruff, H.: Room Acoustics, 4th edn. Spon Press, London (2000)

26. Billon, A., Picaut, J., Sakout, A.: Prediction of the reverberation time in high absorbent room using a modified-diffusion model. Appl. Acoust. 69, 68-74 (2008)
27. Jing, Y., Xiang, N.: On boundary conditions for the diffusion equation in room acoustic prediction: theory, simulations, and experiments. J. Acoust. Soc. Am. 123(1), 145-153 (2008)

28. Visentin, C., Prodi, N., Valeau, V., Picaut, J.: A numerical investigation of the Fick's law of diffusion in room acoustics. J. Acoust. Soc. Am. 132(5), 3180-3189 (2013). https://doi.org/10.1121/1. 4756924

29. Navarro, J.M., Jacobsen, F., Escolano, J., López, J.J.: A theoretical approach to room acoustic simulations based on a radiative transfer model. Acta Acust. United Acust. 96, 1078-1089 (2010). https:// doi.org/10.3813/AAA.918369

30. Navarro, J.M., Escolano, J.: Simulation of building indoor acoustics using an acoustic diffusion equation model. J. Build. Perform. Simul. 8(1), 3-14 (2013). https://doi.org/10.1080/19401493.2013. 850534

31. Brinkmann, F., Aspöck, L., Ackermann, D., Lepa, S., Vorländer, M., Weinzierl, S.: A round robin on room acoustical simulation and auralization. J. Acoust. Soc. Am. 145(4), 2746-2760 (2019)

32. Morse, P., Feshbach, H.: Methods of Theoretical Physics. McGrawHill, New York (1955)

33. Xiang, N., Escolano, J., Navarro, J.M., Jing, Y.: Investigation on the effect of aperture sizes and receiver positions in coupled rooms. J. Acoust. Soc. Am. 133(6), 3975-3985 (2013)

34. Sü Gül, Z., Çalışkan, M., Tavukcuoglu, A., Xiang, N.: Assessment of acoustical indicators in multi-domed historic structures by non-exponential energy decay analysis. Acoust. Aust. 46, 181-192 (2018)

Publisher's Note Springer Nature remains neutral with regard to jurisdictional claims in published maps and institutional affiliations. 